\title{
Propagation of Growth Uncertainty in a Physiologically Structured Population
}

\author{
H.T. Banks *, S. Hu \\ Center for Research in Scientific Computation, Center for Quantitative Sciences in Biomedicine \\ North Carolina State University, Raleigh, NC 27695-8212 USA
}

\begin{abstract}
In this review paper we consider physiologically structured population models that have been widely studied and employed in the literature to model the dynamics of a wide variety of populations. However in a number of cases these have been found inadequate to describe some phenomena arising in certain real-world applications such as dispersion in the structure variables due to growth uncertainty/variability. Prompted by this, we described two recent approaches that have been investigated in the literature to describe this growth uncertainty/variability in a physiologically structured population. One involves formulating growth as a Markov diffusion process while the other entails imposing a probabilistic structure on the set of possible growth rates across the entire population. Both approaches lead to physiologically structured population models with nontrivial dispersion. Even though these two approaches are conceptually quite different, they were found in [17] to have a close relationship: in some cases with properly chosen parameters and coefficient functions, the resulting stochastic processes have the same probability density function at each time.
\end{abstract}

Keywords and phrases: uncertainty, structured population models, Markov diffusion processes, Itô stochastic differential equations, Fokker-Planck equations

Mathematics Subject Classification: 35Q80, 60H10, 60J60, 92D25

\section{Introduction}

In this paper, we give a review of two approaches that have been investigated in the literature (e.g., see $[12,14,17])$ to model growth uncertainty in a physiologically structured population. One approach involves formulating growth as a Markov diffusion process described by Itô stochastic differential equations (described below). This approach is referred to as the stochastic formulation, and it leads to Fokker-Planck physiologically structured population dynamics (defined below). The other approach entails imposing a probabilistic structure on the set of possible growth rates across the entire population. This approach is called the probabilistic formulation, and it leads to growth rate distributed physiologically structured population dynamics (also defined below). While of direct interest to the population modeling investigations, our recent efforts [17] have led in addition to new mathematical understanding of the relationship between the two seemingly disparate modeling approaches. The relationship between these two approaches

\footnotetext{
${ }^{*}$ Corresponding author. E-mail: htbanks@ncsu.edu
} 
is also reviewed in this paper, as is the relationship between the two resulting physiologically structured population models exhibiting dispersion. Before addressing our main task, we shall use this introduction to briefly review some background material. We shall consider canonical types of equations and specific examples of their use in modelling.

During the past half century, physiologically structured population models have played roles of increasing importance, especially in modeling in the biological and life sciences. Indeed there now exist numerous books $[32,40,48,54,64]$ on the subject, most focusing on particular biological applications ranging from age and size structure in populations to tumor models to cell level immunology (where the structure variable is activation level) to viral spread in populations (where the structure variable is immunity level) to recent applications in cell proliferation (where the structure variable is label content). The literature is so extensive as to preclude an exhaustive survey but the recent summaries in $[23,37,62]$ give, for example, a reasonable summary of recent efforts in cell division models. Among other notable recent efforts of interest we mention $[3,4,7-9,13,15,16,18,19,21,22,25,27,28,30,35,37,44-46,57]$. Many of these efforts involve deterministic modeling efforts whereas here we focus on a class of structure population models involving growth uncertainty.

Modeling in the presence of uncertainty has been a great interest to researchers for some decades. Especially in recent years, due to the vast applications of mathematical modeling in biology and clinical research studies, it has become increasingly evident that properly incorporating randomness into a mathematical model can answer many questions that deterministic models can not. In this paper, we consider incorporating randomness into the following system of ordinary differential equations

$$
\dot{\mathbf{x}}(t)=\mathbf{g}(t, \mathbf{x}(t)), \quad \mathbf{x}(0)=\mathbf{x}_{0},
$$

where $\mathbf{x}=\left(x_{1}, x_{2}, \ldots, x_{n}\right)^{T}, \mathbf{g}=\left(g_{1}, g_{2}, \ldots, g_{n}\right)^{T}$ is $n$-dimensional nonrandom functions of $t$ and $\mathbf{x}$, and $\mathbf{x}_{0}$ is a $n$-dimensional column vector. For our application here (1.1) is used to describe the growth process of an individual, where $\mathbf{x}$ is interpreted as structure variables, $\mathbf{g}$ is the vector of growth rate functions, and $\mathbf{x}_{0}$ is the initial structure level of this individual.

The simplest way to incorporate randomness into (1.1) is through the initial condition (that is, only the initial condition is random), and the resulting equation is given by

$$
\dot{\mathbf{x}}\left(t ; \mathbf{X}_{0}\right)=\mathbf{g}\left(t, \mathbf{x}\left(t ; \mathbf{X}_{0}\right)\right), \quad \mathbf{x}\left(0 ; \mathbf{X}_{0}\right)=\mathbf{X}_{0},
$$

where $\mathbf{X}_{0}$ is a $n$-dimensional random vector. Note that for each $t, \mathbf{x}\left(t ; \mathbf{X}_{0}\right)$ is a function of the random variables $\mathbf{X}_{0}$ and thus is also an $n$-dimensional random variable. Hence $\left\{\mathbf{x}\left(t ; \mathbf{X}_{0}\right)\right\}$ is a stochastic process.

Equation (1.2) is often referred to as a cryptodeterministic equation (e.g., see [33,49,60]) since the random initial vector $\mathbf{X}_{0}$ is propagated deterministically. Cryptodeterministic equations have proven useful in a wide range of applications such as classical statistical mechanics, statistical thermodynamics, kinetic theory and biosciences (e.g., see [60, Chapter 6] for more information). For our application here the cryptodeterministic equation (1.2) is used to describe the growth processes of individuals in a population in which all the individuals follow the same growth rate functions $\mathbf{g}$, even though they may start with different initial structure levels.

The probability density function One method of treating some suitable types of stochastic process $\mathbf{X}(t)$ (such as the one obtained with the cryptodeterministic equation (1.2)) is to obtain equations describing the time evolution of probability density function $p(t, \mathbf{x})$ of $\mathbf{X}(t)$ (interested readers may refer to [67] for a general introduction on probability density functions). In such cases, $p(t, \mathbf{x})$ generally satisfies a partial differential equation. The resulting partial differential equations are often found to have their own wide applications. For example, as we shall see below, the resulting time-evolution equation for $p(t, \mathbf{x})$ of the solution $\mathbf{X}(t)=\mathbf{x}\left(t ; \mathbf{X}_{0}\right)$ to the cryptodeterministic equation (1.2) is a special case of some classical physiological structured population models. Hence, this conceptual approach is the manner in which we view the stochastic process obtained by the cryptodeterministic equation, and it is also the one that we use to treat the stochastic processes obtained by both our stochastic and our probabilistic formulations. 


\subsection{The Liouville Equation}

The time-evolution equation for the probability density function of stochastic process resulting from a cryptodeterministic equation (1.2) is stated in the following so-called Liouville's "theorem" (e.g., see [60, Theorem 6.2.2]).

Theorem 1.1. Assume that $\mathbf{X}_{0}$ has probability density function $p_{0}$, and (1.2) has a mean square solution $\mathbf{x}\left(t ; \mathbf{X}_{0}\right)$. Then the probability density function $p(t, \mathbf{x})$ of the solution $\mathbf{X}(t)=\mathbf{x}\left(t ; \mathbf{X}_{0}\right)$ satisfies

$$
\frac{\partial}{\partial t} p(t, \mathbf{x})+\sum_{k=1}^{n} \frac{\partial}{\partial x_{k}}\left(g_{k}(t, \mathbf{x}) p(t, \mathbf{x})\right)=0
$$

with initial condition $p(0, \mathbf{x})=p_{0}(\mathbf{x})$.

The resulting equation (1.3) is often called Liouville's equation (this is a key equation-actually a conservation principle-in statistical mechanics and statistical thermodynamics [39]), and it includes the equation for describing the evolution of the probability density function for a conservative Hamiltonian system (e.g., see [39, Section 4.2], [49, Section 1.3]) as special case.

\subsection{Classical Physiologically Structured Population Models}

We observe that the Liouville equation (1.3) with $g_{1} \equiv 1$ is a special case of a physiologically structured population model given by Oster and Takahashi in 1974 [53]

$$
\frac{\partial}{\partial t} u(t, \mathbf{x})+\frac{\partial}{\partial x_{1}} u(t, \mathbf{x})+\sum_{j=2}^{n} \frac{\partial}{\partial x_{j}}\left(g_{j}(t, \mathbf{x}) u(t, \mathbf{x})\right)+d(t, \mathbf{x}) u(t, \mathbf{x})=0
$$

without mortality (i.e., $d \equiv 0$ ). This model is used to describe the population density $u(t, \mathbf{x})$ (number of individuals per unit structure level) in a spatially homogeneous population where individuals are characterized by structure variables $\mathbf{x}=\left(x_{1}, x_{2}, \cdots, x_{n}\right)^{T}$ with $x_{1}$ denoting the chronological age and $x_{2}, \cdots, x_{n}$ representing some physiological variables such as mass, volume, chemical composition, and other quantities having an influence on an individual's growth and mortality rates, $\left(g_{2}(t, \mathbf{x}), g_{3}(t, \mathbf{x}), \ldots, g_{n}(t, \mathbf{x})\right)^{T}$ is the vector for the growth rates of individuals with structure variable $\mathbf{x}$ at time $t$, and $d(t, \mathbf{x})$ is the mortality rate of individuals with structure variable $\mathrm{x}$ at time $t$.

Equation (1.4) includes a number of well-known structured population models as special cases. Specifically, the model with only chronological age and death involved, that is,

$$
\frac{\partial}{\partial t} u(t, x)+\frac{\partial}{\partial x} u(t, x)+d(t, x) u(t, x)=0,
$$

is the age-structured population model given by Mckendrick [47] in 1926 and Von Foerster [63] in 1959. Equation (1.4) with $n=2$, i.e.,

$$
\frac{\partial}{\partial t} u(t, \mathbf{x})+\frac{\partial}{\partial x_{1}} u(t, \mathbf{x})+\frac{\partial}{\partial x_{2}}\left(g_{2}(t, \mathbf{x}) u(t, \mathbf{x})\right)+d(t, \mathbf{x}) u(t, \mathbf{x})=0,
$$

is the age-size structured population model developed by Sinko and Streifer [59] in 1967 (with $x_{2}$ being some physiological variable), and it is also the model given by Bell and Anderson [27] in 1967 for the cell population (with $x_{2}$ denoting the volume of the cell). Equation (1.6) without the second term, that is,

$$
\frac{\partial}{\partial t} u(t, x)+\frac{\partial}{\partial x}(g(t, x) u(t, x))+d(t, x) u(t, x)=0,
$$

is often referred to as Sinko-Streifer model or size-structured population model. Here $x$ is the structure variable, which may represent weight, length, volume, chronological age (in this latter case, equation 
(1.7) becomes the age-structured population model (1.5)), caloric content, label intensity, etc, depending on the applications.

General features Based on above discussions, we see that all these physiologically structured population models are built on the assumption that individuals with the same structure level at same time have the same growth rates, and the only uncertainty enters the population is through the initial data (i.e., uncertainty is due to the variability in the initial structure level of individuals across the population). These structured population models with proper boundary and initial conditions along with their corresponding nonlinear versions have been widely studied in the literature for both computational and theoretical analysis including well-posedness studies, asymptotic analysis, sensitivity analysis, parameter estimation techniques and control studies. In addition, they have been used to model the dynamics of a wide variety of populations such as cells, plants and marine species (e.g., [7,21,48,55] and the references therein). However, they are not adequate to describe phenomena such as dispersion presented in some real-world application problems. For example, the experimental data in $[12,13]$ for the early growth of shrimp reveals that juvenile shrimp populations exhibit a great deal of variability in size as time evolves even though the shrimp begin with approximately similar sizes. This phenomenon was also observed in studies $[8,16]$ of mosquitofish growth in rice fields, where the data exhibit both dispersion and bimodality in size as time increases (without reproduction involved). Thus for these applications a cryptodeterministic formulation (1.3) is not adequate to describe the growth of individuals across the population, and one needs to incorporate some type of uncertainty/variability into the growth process of individuals in addition to any variability in the initial structure level of individuals across the population.

\section{Stochastic and Probabilistic Formulations and Their Resulting Physiologically Structured Models}

In this section, we consider another two ways (one stochastic and one probabilistic in nature) to incorporate randomness into system of ordinary differential equations (1.1) to model growth uncertainty in a physiologically structured population. As we shall see below, both approaches result in physiologically structured population models with dispersion.

\subsection{Stochastic Formulation and Its Associated Fokker-Planck Physiologically Structured Population Models}

The stochastic formulation is motivated by recognizing that some local factors such as environmental or emotional fluctuations can have a significant influence on the individual growth. For example, the growth rates of shrimp are affected by several environmental factors such as temperature, dissolved oxygen level and salinity. Our stochastic formulation is constructed based on the assumption that growth process of each individual can be described by a Markov diffusion process. Thus, for this formulation the uncertainty is introduced into the entire population by the growth uncertainty of each individual as well as the variability in the initial structure level of individuals across the population.

We begin with a Markov diffusion process $\mathbf{X}(t)$ described by the system of stochastic differential equations

$$
d \mathbf{X}(t)=\mathbf{g}(t, \mathbf{X}(t)) d t+\boldsymbol{\sigma}(t, \mathbf{X}(t)) d \mathbf{W}(t), \quad \mathbf{X}(0)=\mathbf{X}_{0},
$$

where $\mathbf{X}=\left(X_{1}, X_{2}, \ldots, X_{n}\right)^{T}, \mathbf{g}=\left(g_{1}, g_{2}, \ldots, g_{n}\right)^{T}$ is a nonrandom $n$-dimensional function of $t$ and $\mathbf{x}, \boldsymbol{\sigma}$ is a nonrandom $n \times l$ matrix function of $t$ and $\mathbf{x}$, and $\mathbf{W}(t)$ is an $l$-dimensional standard Wiener process independent of the random initial vector $\mathbf{X}_{0}$. We recall that stochastic differential equations can be interpreted in either an Itô or Stratonovich sense (e.g., see [33, Chapter 3] for details). In this paper, we assume that the stochastic differential equations are interpreted in the Itô sense.

Definition 2.1. The stochastic differential equation interpreted in an Itô sense is referred to as an Itô stochastic differential equation. For the system of Itô stochastic differential equations (2.1), $\mathbf{g}$ is called the drift coefficient, and $\boldsymbol{\Sigma}=\boldsymbol{\sigma} \boldsymbol{\sigma}^{T}$ is known as the diffusion coefficient. 
For our application here the realization $\mathbf{x}$ of $\mathbf{X}(t)$ at time $t$ represents structure level, and $\mathbf{g}(t, \mathbf{x})$ and $\boldsymbol{\Sigma}(t, \mathbf{x})$ denote the mean growth rates and the variance in the growth rates, respectively, of individuals with structure variables $\mathbf{x}$ at time $t$. We note that when $\boldsymbol{\sigma}$ vanishes, (2.1) reduces to the cryptodeterministic equation (1.3).

\subsubsection{Probability Density Function of $\mathbf{X}(t)$}

We assume that $\mathbf{g}$ and $\boldsymbol{\sigma}$ satisfy conditions that guarantee the existence and uniqueness of solutions (e.g., see [42, Theorem 6.22] or [51, Theorem 5.2.1] for details) to the initial value problem (2.1). We further assume that the functions involved in this section are sufficiently smooth so that all the differentiations carried out make sense. With these assumptions the time-evolution equation for the probability density function of stochastic process resulting from the system of Itô stochastic differential equations (2.1) is stated in the following theorem (e.g., see [34, Section 5.2], [49, Section 8.1] or [52, Section 5.3.2]).

Theorem 2.2. Assume that $\mathbf{X}_{0}$ has probability density function $p_{0}$, and $\mathbf{X}(t)$ satisfies $(2.1)$. Then the probability density function $p(t, \mathbf{x})$ of $\mathbf{X}(t)$ satisfies

$$
\frac{\partial}{\partial t} p(t, \mathbf{x})+\sum_{k=1}^{n} \frac{\partial}{\partial x_{k}}\left(g_{k}(t, \mathbf{x}) p(t, \mathbf{x})\right)=\frac{1}{2} \sum_{k, j=1}^{n} \frac{\partial^{2}}{\partial x_{k} \partial x_{j}}\left[\Sigma_{k j}(t, \mathbf{x}) p(t, \mathbf{x})\right]
$$

with initial condition $p(0, \mathbf{x})=p_{0}(\mathbf{x})$. Here $\Sigma_{k j}(t, \mathbf{x})$ is the $(k, j)$ th element of the matrix $\boldsymbol{\Sigma}(t, \mathbf{x})$.

The resulting equation (2.2) is called the Fokker-Planck equation or Kolmogorov's forward equation. The Fokker-Planck equation arises often in and is important to the fields of chemistry and physics (e.g., see [56]). In an early biological application [41], a scalar Fokker-Planck equation was employed to study the random fluctuation of gene frequencies in natural population (where $x$ denotes gene frequency). The Fokker-Planck equation has also been effectively used in the literature (e.g., see [26] and the reference therein) to model the dispersal behavior of a population such as studies of female cabbage root fly movement in the presence of Brassica odors, and movement of flea beetles in cultivated collard patches (in these cases $\mathbf{x}$ denotes the space position and $g(t, \mathbf{x})$ is the mean velocity of individuals at position $\mathbf{x}$ at time $t)$.

\subsubsection{Fokker-Planck Physiologically Structured Population Models}

A more general case of the Fokker-Planck equation (2.2) with mortality is given by

$$
\frac{\partial}{\partial t} u(t, \mathbf{x})+\sum_{k=1}^{n} \frac{\partial}{\partial x_{k}}\left(g_{k}(t, \mathbf{x}) u(t, \mathbf{x})\right)+d(t, \mathbf{x}) u(t, \mathbf{x})=\frac{1}{2} \sum_{k, j=1}^{n} \frac{\partial^{2}}{\partial x_{k} \partial x_{j}}\left[\Sigma_{k j}(t, \mathbf{x}) u(t, \mathbf{x})\right],
$$

where $u(t, \mathbf{x})$ represents population density of individuals with structure levels $\mathbf{x}$ at time $t$, and $d(t, \mathbf{x})$ denotes the mortality rate of individuals with structure levels $\mathbf{x}$ at time $t$. Actually (2.3) is associated with a so-called "killed diffusion" process $\tilde{\mathbf{X}}(t)$ defined by

$$
\tilde{\mathbf{X}}(t)= \begin{cases}\mathbf{X}(t), & \text { if } t<K_{T} \\ \Delta, & \text { if } t \geq K_{T}\end{cases}
$$

where $K_{T}$ is the "killing time" with killing rate $d(t, \mathbf{x})$, and $\Delta$ is the "coffin" (or "cemetery") state which is not in $\mathbb{R}^{n}$ (e.g., see [43, Section 5.7] for details).

Equation (2.3) includes a number of structured population models as special cases. Specifically, (2.3) with $g_{1} \equiv 1$ and all the elements of the matrix $\boldsymbol{\Sigma}(t, \mathbf{x})$ being zero except the diagonal elements $\Sigma_{k k}$, $k=2, \ldots, n$ is the physiologically structured population model given by Oster and Takahashi [53] in 1974

$$
\begin{array}{r}
\frac{\partial}{\partial t} u(t, \mathbf{x})+\frac{\partial}{\partial x_{1}} u(t, \mathbf{x})+\sum_{k=2}^{n} \frac{\partial}{\partial x_{k}}\left(g_{k}(t, \mathbf{x}) u(t, \mathbf{x})\right) \\
+d(t, \mathbf{x}) u(t, \mathbf{x})=\frac{1}{2} \sum_{k=2}^{n} \frac{\partial^{2}}{\partial x_{k}^{2}}\left[\Sigma_{k k}(t, \mathbf{x}) u(t, \mathbf{x})\right]
\end{array}
$$


where individuals are characterized by the chronological age $x_{1}$, and physiological variables $x_{2}, \ldots, x_{n}$ whose growth rates are affected by various stochastic factors. Observe that (2.5) with $n=2$, i.e.,

$$
\frac{\partial}{\partial t} u(t, \mathbf{x})+\frac{\partial}{\partial x_{1}} u(t, \mathbf{x})+\frac{\partial}{\partial x_{2}}\left(g_{2}(t, \mathbf{x}) u(t, \mathbf{x})\right)+d(t, \mathbf{x}) u(t, \mathbf{x})=\frac{1}{2} \frac{\partial^{2}}{\partial x_{2}^{2}}\left[\Sigma_{22}(t, \mathbf{x}) u(t, \mathbf{x})\right],
$$

is a model given by Weiss [66] in 1968 for cell populations with age structure.

In this paper equation (2.3) with appropriate boundary and initial conditions along with its special cases with nonzero diffusion coefficients such as (2.2), (2.5) and (2.6) will be referred to as Fokker-Planck physiologically structured (FPPS) population models. In light of the above discussions, we see that FPPS population models can be associated with a stochastic process resulting from the stochasticity of growth rates of each individual along with the variability in the initial structure level of individuals across the population.

Fokker-Planck physiologically structured population models have been investigated by a number of researchers. For example, a scalar FPPS model with nonlinear and nonlocal boundary condition was studied in [31] to understand how a constant diffusion coefficient influences the stability and the Hopf bifurcation of the positive equilibrium of the system. In another example inverse problems were considered in [26] for the estimation of temporally and spatially varying coefficients in a scalar FPPS model with nonlocal boundary conditions.

\subsection{Probabilistic Formulation and Its Associated Growth Rate Distributed Physiologically Structured Population Models}

Our probabilistic formulation is motivated by the observation that some intrinsic variables (e.g., genetic differences or non-lethal infections due to chronic disease) can have an effect on individual growth. For example, in many marine species such as mosquitofish, females grow faster than males, which means that individuals even with the same size may have different growth rates. In addition, it was reported in [30] that non-lethal infection of Penaeus vannamei postlarvae by IHHNV (infectious hypodermal and hematopoietic Necrosis virus) may reduce growth and increase size variability.

The underlying assumption for the probabilistic formulation is that each individual grows according to a deterministic growth model, but different individuals may have different structure dependent growth rates. Based on this assumption, one partitions the entire population into (possibly a continuum of) subpopulations where individuals in each subpopulation have the same structure-dependent growth rates, and then assigns a probability distribution to this partition of possible growth rates in the population. Hence, for this formulation the uncertainty is introduced into population by the variability of growth rates among subpopulations as well as possible variability in the initial structure level of individuals across population.

For simplicity in explanation, we assume that growth processes of individuals are characterized by the following system of differential equations

$$
\dot{\mathbf{x}}\left(t ; \mathbf{X}_{0}, \mathbf{Z}\right)=\mathbf{g}\left(t, \mathbf{x}\left(t ; \mathbf{X}_{0}, \mathbf{Z}\right) ; \mathbf{Z}\right), \quad \mathbf{x}\left(0 ; \mathbf{X}_{0}, \mathbf{Z}\right)=\mathbf{X}_{0}
$$

where $\mathbf{x}=\left(x_{1}, x_{2}, \ldots, x_{n}\right)^{T}, \mathbf{g}=\left(g_{1}, g_{2}, \ldots, g_{n}\right)^{T}$ is an $n$-dimensional nonrandom function of $t$ and $\mathbf{x}, \mathbf{Z}$ is an $m$-dimensional random vector (either discrete or continuous) that is used to account for the variability in growth, and $\mathbf{X}_{0}$ is an $n$-dimensional random vector used to account for the variability among individuals in the initial structure level. Equation (2.7) implies that all the individuals have the same functional form $\mathbf{g}(t, \mathbf{x} ; \mathbf{z})$ for the growth rates but the values of parameters $\mathbf{z}$ may vary across individuals, where $\mathbf{z}$ is realization of $\mathbf{Z}$. To differentiate it from the system of stochastic differential equations discussed in the above section, in this paper (2.7) is referred to as a system of random differential equations (a term popularized for some years since its early use in $[60,61])$. 


\subsubsection{Probability Density Function of $\mathbf{x}\left(t ; \mathbf{X}_{0}, \mathbf{Z}\right)$}

Note that for each $t, \mathbf{x}\left(t ; \mathbf{X}_{0}, \mathbf{Z}\right)$ is a function of the random variables $\mathbf{X}_{0}$ and $\mathbf{Z}$ and thus is also a random variable. Hence $\left\{\mathbf{x}\left(t ; \mathbf{X}_{0}, \mathbf{Z}\right)\right\}$ is a stochastic process. We observe that for any given value $\mathbf{z}$ of $\mathbf{Z}$ system (2.7) is cryptodeterministic. Hence, by using the Liouville equation (1.3) the probability density function $\varphi(t, \mathbf{x} ; \mathbf{z})$ of solution $\mathbf{x}\left(t ; \mathbf{X}_{0}, \mathbf{z}\right)$ to $(2.7)$ with given value $\mathbf{z}$ of $\mathbf{Z}$ satisfies

$$
\frac{\partial}{\partial t} \varphi(t, \mathbf{x} ; \mathbf{z})+\sum_{k=1}^{n} \frac{\partial}{\partial x_{k}}\left(g_{k}(t, \mathbf{x} ; \mathbf{z}) \varphi(t, \mathbf{x} ; \mathbf{z})\right)=0 .
$$

Given the conditional probability density function $\varphi(t, \mathbf{x} ; \mathbf{z})$, the probability density function $p(t, \mathbf{x})$ of $\mathbf{x}\left(t ; \mathbf{X}_{0}, \mathbf{Z}\right)$ is given by

$$
p(t, \mathbf{x})=\int_{\mathbb{Z}} \varphi(t, \mathbf{x} ; \mathbf{z}) d \Psi(\mathbf{z}),
$$

where $\mathbb{Z}$ denotes the set of all possible values of $\mathbf{Z}$, and $\Psi(\mathbf{z})$ denotes the cumulative distribution function of $\mathbf{Z}$. It should be noted that for the discrete case, the associated probability density function $\psi$ of $\mathbf{Z}$ can be written in terms of Dirac delta function. For example, if the discrete random vector $\mathbf{Z}$ can take $l$ different values $\mathbf{z}_{j}, j=1,2, \ldots, l$, then its associated probability density function $\psi$ can be written as

$$
\psi(\mathbf{z})=\sum_{j=1}^{l} \beta_{j} \delta\left(\mathbf{z}-\mathbf{z}_{j}\right) .
$$

Here $\delta$ denotes the Dirac delta function, $\beta_{j}$ is the probability associated with $\mathbf{z}_{j}, j=1,2, \ldots, l$, and $\sum_{j=1}^{l} \beta_{j}=1$. Thus, for this case (2.9) can be simply written as follows

$$
p(t, \mathbf{x})=\sum_{j=1}^{l} \beta_{j} \varphi\left(t, \mathbf{x} ; \mathbf{z}_{j}\right) .
$$

\subsubsection{Growth Rate Distributed Physiologically Structured Population Models}

A more general case of (2.8) including mortality is given by

$$
\frac{\partial}{\partial t} v(t, \mathbf{x} ; \mathbf{z})+\sum_{j=1}^{n} \frac{\partial}{\partial x_{j}}\left(g_{j}(t, \mathbf{x} ; \mathbf{z}) v(t, \mathbf{x} ; \mathbf{z})\right)+d(t, \mathbf{x}) v(t, \mathbf{x} ; \mathbf{z})=0
$$

where $v(t, \mathbf{x} ; \mathbf{z})$ denotes the population density of individuals with structure level $\mathbf{x}$ at time $t$ in a subpopulation with growth rate $\mathbf{g}(t, \mathbf{x} ; \mathbf{z})$, and $d(t, \mathbf{x})$ denotes mortality rate of individuals with structure level $\mathbf{x}$ at time $t$ (for simplicity here we assume that all individuals have the same time and structure level dependent mortality rate - this can be readily extended to include a distribution for mortality rates). Given the subpopulation density $v(t, \mathbf{x} ; \mathbf{z})$, the population density $u(t, \mathbf{x})$ for the population with structure level $\mathbf{x}$ at time $t$ is given by

$$
u(t, \mathbf{x})=\int_{\mathbb{Z}} v(t, \mathbf{x} ; \mathbf{z}) d \Psi(\mathbf{z}) .
$$

In this paper equation (2.12) along with (2.11) (with appropriate boundary and initial conditions) as well as its special cases (e.g., the model with zero mortality) are referred to as growth rate distributed physiologically structured (GRDPS) population models. This GRDPS model was originally developed and studied in $[8,15]$ in connection with mosquito fish population, where it was called the growth rate distribution model and it was not formulated directly from a stochastic process. However, from the above discussions, it can be readily seen that GRDPS models in fact are associated with the stochastic process 
$\mathbf{x}\left(t ; \mathbf{X}_{0}, \mathbf{Z}\right)$, which is obtained through the variability $\mathbf{Z}$ in the individual's growth rates along with the variability $\mathbf{X}_{0}$ in the initial structure level of individuals in the population.

We observe that the GRDPS model involves a stationary probabilistic structure on a family of deterministic dynamical systems. For the case where $\mathbf{Z}$ is a discrete random vector with associated probability density function described by (2.10), the GRDPS model is comprised of $l$ classical physiological structured population models with each model describing the dynamics of one subpopulation. This is the case that is often investigated in the literature (e.g., $[1,23-25,37,58])$ to account for the heterogeneity of the population. For example, to account for the distinct cell proliferation properties among cells, the authors in $[23,24,58]$ divide the cell population into a finite number of subpopulations based on the cell types and the number of cell divisions.

In practice, the distribution function $\Psi$ on the growth rates is the fundamental "parameter" to be determined from aggregate data (e.g., sampling with or without replacement from the entire population) through either parametric methods (which depend on prior information known about the form for $\Psi$ ) or nonparametric methods (used in cases where one has no knowledge of the form for $\Psi$ ). Several methods (e.g., a delta function based method and a piecewise linear spline based method) in the nonparametric approach have been proposed and successfully implemented in the literature (e.g., $[6,10,11,15,16,20]$ and the references therein). These methods are based on the Prohorov [50] metric theoretical framework developed earlier in [6] and described more fully in [5] to estimate the growth rate distribution function $\Psi$; the estimation accuracy between these two methods was compared in [10].

A growth rate distributed physiological structured population model has been successfully used to model mosquitofish population in the rice fields, where the experimental data exhibits both bimodality and dispersion in size as time increases (e.g., see $[8,15,16]$ ) even though the population begins with a unimodal density (no reproduction involved). In addition, this model was also used to model the early growth of shrimp populations [12,13], which exhibit a great deal of variability in size as time evolves even though the shrimp begin with approximately similar sizes (i.e., essentially very little to no variance in initial size structure).

\section{A Relationship Between Stochastic and Probabilistic Formulations as well as a Relationship Between the FPPS and GRDPS Models}

Our formulations in Section 2 make clear that the stochastic and probabilistic formulations are conceptually quite different in describing the growth process of individuals with one depicted by an Itô stochastic differential equation and the other by random differential equation. Each formulation leads to an underlying stochastic process and a natural question is how these two processes might differ. Somewhat surprisingly however, it was shown in [17] that there are a class of Itô stochastic differential equations (SDEs) for which their solutions have the same probability density functions at each time $t$ as those for the solutions of their corresponding random differential equations (RDEs) formulations.

Definition 3.1. If the probability density function of the solution of an SDE is the same as that of the solution of an RDE at each time $t$, then the SDE and RDE are said to be pointwise equivalent.

It should be noted that even though stochastic differential equations and their corresponding random differential equations can yield pointwise equivalent densities, the stochastic processes obtained by these SDEs and their corresponding RDEs are in general different as their covariance functions need not be the same (see [17] for details with examples).

In the next four subsections, we summarize the pointwise equivalence results from [17], where it was shown how to transform from a given SDE to the corresponding equivalent RDE, and from a given RDE to the corresponding SDE. Since the SDE can lead to a computationally challenging system (e.g., when convection dominated diffusion is involved) while the RDE formulation leads to a system that is readily solved with eminently parallelizable computational methods (see the remarks below and $[16,17]$ for further discussions), the numerical advantages for an alternate formulation for the associated FokkerPlanck equations are rather obvious. 


\subsection{Scalar Affine Differential Equations (Class 1)}

In the first class of systems, the random differential equation considered has the following form

$$
\frac{d x\left(t ; X_{0}, \mathbf{Z}\right)}{d t}=\alpha(t) x\left(t ; X_{0}, \mathbf{Z}\right)+\gamma(t)+\mathbf{Z} \cdot \varrho(t), \quad x\left(0 ; X_{0}, \mathbf{Z}\right)=X_{0} .
$$

In the above equation, $\mathbf{Z}=\left(Z_{0}, Z_{1}, \ldots, Z_{m-1}\right)^{T}$, where $Z_{j} \sim \mathcal{N}\left(\mu_{j}, \sigma_{j}^{2}\right), j=0,1,2, \ldots, m-1$, are mutually independent with $\mu_{j}, \sigma_{j}, j=0,1,2, \ldots, m-1$, being some positive constants. In addition, $\alpha$, $\gamma$ and $\varrho=\left(\varrho_{0}, \varrho_{1}, \ldots, \varrho_{m-1}\right)^{T}$ are non-random functions of $t$. The corresponding stochastic differential equation takes the form

$$
d X(t)=[\alpha(t) X(t)+\xi(t)] d t+\eta(t) d W(t), \quad X(0)=X_{0},
$$

with $\xi$ and $\eta$ being some non-random functions of $t$.

The conditions for the pointwise equivalence between random differential equation (3.1) and stochastic differential equation (3.2) are stated in the following theorem (see [17] for a proof).

Theorem 3.2. If functions $\xi, \eta$ and $\gamma$, and functions $\varrho_{j}$ and constants $\mu_{j}$ and $\sigma_{j}, j=0,1,2, \ldots, m-1$ satisfy the following two equalities

$$
\int_{0}^{t} \xi(s) \exp \left(\int_{s}^{t} \alpha(\tau) d \tau\right) d s=\int_{0}^{t}[\gamma(s)+\boldsymbol{\mu} \cdot \varrho(s)] \exp \left(\int_{s}^{t} \alpha(\tau) d \tau\right) d s
$$

and

$$
\int_{0}^{t}\left[\eta(s) \exp \left(\int_{s}^{t} \alpha(\tau) d \tau\right)\right]^{2} d s=\sum_{j=0}^{m-1} \sigma_{j}^{2}\left[\int_{0}^{t} \varrho_{j}(s) \exp \left(\int_{s}^{t} \alpha(\tau) d \tau\right) d s\right]^{2},
$$

then random differential equation (3.1) and stochastic differential equation (3.2) yield stochastic processes that are pointwise equivalent. Here $\boldsymbol{\mu}=\left(\mu_{0}, \mu_{1}, \ldots, \mu_{m-1}\right)^{T}$.

RDE to SDE Based on equivalence conditions (3.3) and (3.4), the specific form of the corresponding pointwise equivalent SDE can be given in terms of the known RDE. To do that, we assume that $\varrho$ has the property that the function

$$
h(t)=\sum_{j=0}^{m-1} \sigma_{j}^{2} \varrho_{j}(t) \int_{0}^{t} \varrho_{j}(s) \exp \left(\int_{s}^{t} \alpha(\tau) d \tau\right) d s
$$

is nonnegative for any $t \geq 0$. By the equivalent conditions (3.3) and (3.4), the corresponding pointwise equivalent $\mathrm{SDE}$ for RDE (3.1) is then given by

$$
d X(t)=[\alpha(t) X(t)+\gamma(t)+\boldsymbol{\mu} \cdot \varrho(t)] d t+\sqrt{2 h(t)} d W(t), \quad X(0)=X_{0}
$$

SDE to RDE We consider the conversion from SDE to equivalent RDE. The equivalence conditions (3.3) and (3.4) imply that there are numerous different choices for the RDE (through choosing different values for $m$ ). One of the simple choices of the corresponding pointwise equivalent RDE for the SDE (3.2) is given by

$$
\frac{d x\left(t ; X_{0}, Z\right)}{d t}=\alpha(t) x\left(t ; X_{0}, Z\right)+\xi(t)+Z \varrho_{0}(t), \quad x\left(0 ; X_{0}, Z\right)=X_{0},
$$

where the random variable $Z \sim \mathcal{N}(0,1)$, and the function $\varrho_{0}$ is defined by

$$
\varrho_{0}(t)=\frac{d}{d t}\left[\left(\int_{0}^{t} \eta^{2}(s) \exp \left(2 \int_{s}^{t} \alpha(\tau) d \tau\right) d s\right)^{\frac{1}{2}}\right]-\alpha(t)\left[\int_{0}^{t} \eta^{2}(s) \exp \left(2 \int_{s}^{t} \alpha(\tau) d \tau\right)\right]^{\frac{1}{2}} .
$$

The interested readers can refer to [17] for other possible choices of equivalent RDE. 


\subsection{Scalar Affine Differential Equations (Class 2)}

In this case, the random differential equation considered has the following form

$$
\frac{d x\left(t ; X_{0}, \mathbf{Z}\right)}{d t}=(\mathbf{Z} \cdot \varrho(t)+\gamma(t))\left(x\left(t ; X_{0}, \mathbf{Z}\right)+c\right), \quad x\left(0 ; X_{0}, \mathbf{Z}\right)=X_{0} .
$$

In the above equation, $\mathbf{Z}=\left(Z_{0}, Z_{1}, \ldots, Z_{m-1}\right)^{T}$, where $Z_{j} \sim \mathcal{N}\left(\mu_{j}, \sigma_{j}^{2}\right), j=0,1,2, \ldots, m-1$, are mutually independent with $\mu_{j}, \sigma_{j}, j=0,1,2, \ldots, m-1$, being some positive constants. In addition, $\varrho=\left(\varrho_{0}, \varrho_{1}, \ldots, \varrho_{m-1}\right)^{T}$ is a non-random vector function of $t, \gamma$ is a non-random function of $t$, and $c$ is a given constant. The equivalent stochastic differential equation takes the form

$$
d X(t)=\xi(t)(X(t)+c) d t+\eta(t)(X(t)+c) d W(t), \quad X(0)=X_{0},
$$

where $\xi$ and $\eta$ are some non-random functions of $t$.

The conditions for the pointwise equivalence between random differential equation (3.5) and stochastic differential equation (3.6) are stated in the following theorem (again see [17] for a proof).

Theorem 3.3. If the functions $\xi, \eta$ and $\gamma$, and the function $\varrho_{j}$ and the constants $\mu_{j}$ and $\sigma_{j}, j=$ $0,1,2, \ldots, m-1$, satisfy the following two equalities

$$
\int_{0}^{t}\left(\xi(s)-\frac{1}{2} \eta^{2}(s)\right) d s=\int_{0}^{t}(\boldsymbol{\mu} \cdot \boldsymbol{\varrho}(s)+\gamma(s)) d s
$$

and

$$
\int_{0}^{t} \eta^{2}(s) d s=\sum_{j=0}^{m-1} \sigma_{j}^{2}\left(\int_{0}^{t} \varrho_{j}(s) d s\right)^{2},
$$

then the random differential equation (3.5) and the stochastic differential equation (3.6) yield stochastic processes that are pointwise equivalent. Here $\boldsymbol{\mu}=\left(\mu_{0}, \mu_{1}, \ldots, \mu_{m-1}\right)^{T}$.

RDE to SDE Based on equivalence conditions (3.7) and (3.8), the specific form of the corresponding pointwise equivalent SDE can be given in terms of a known RDE. To do that, we assume that $\varrho$ has the property that the function

$$
\tilde{h}(t)=\sum_{j=0}^{m-1} \sigma_{j}^{2} \varrho_{j}(t)\left(\int_{0}^{t} \varrho_{j}(s) d s\right)
$$

is nonnegative for any $t \geq 0$. Then the corresponding pointwise equivalent $\mathrm{SDE}$ for the RDE (3.5) is given by

$$
d X(t)=(\boldsymbol{\mu} \cdot \varrho(t)+\gamma(t)+\tilde{h}(t))(X(t)+c) d t+\sqrt{2 \tilde{h}(t)}(X(t)+c) d W(t), \quad X(0)=X_{0} .
$$

SDE to RDE The equivalence conditions (3.7) and (3.8) imply that there are many different choices for an equivalent RDE (through choosing different values for $m$ ). One of the simple choices for the corresponding pointwise equivalent RDE for the $\mathrm{SDE}(3.6)$ is as follows:

$$
\frac{d x\left(t ; X_{0}, Z\right)}{d t}=\left(Z \varrho_{0}(t)+\xi(t)-\frac{1}{2} \eta^{2}(t)\right)\left(x\left(t ; X_{0}, Z\right)+c\right), \quad x\left(0 ; X_{0}, Z\right)=X_{0}
$$

where the random variable $Z \sim \mathcal{N}(0,1)$, and the function $\varrho_{0}$ is defined by

$$
\varrho_{0}(t)=\frac{d}{d t}\left[\left(\int_{0}^{t} \eta^{2}(s) d s\right)^{\frac{1}{2}}\right] .
$$

Interested readers can refer to [17] for other possible choices for the equivalent RDE. 


\subsection{Vector Affine Systems}

In this class of systems, we consider vector random differential equations of the form

$$
\frac{d \mathbf{x}\left(t ; \mathbf{X}_{0}, \mathbf{Z}\right)}{d t}=\mathcal{A}(t) \mathbf{x}\left(t ; \mathbf{X}_{0}, \mathbf{Z}\right)+\gamma(t)+\mathcal{H}(t) \mathbf{Z}, \quad \mathbf{x}\left(0 ; \mathbf{X}_{0}, \mathbf{Z}\right)=\mathbf{X}_{0} .
$$

Here $\mathbf{Z}$ is an $m$-dimensional random vector that is a multivariate normal distributed with mean vector $\boldsymbol{\mu}_{\mathbf{Z}}$ and covariance matrix $\boldsymbol{\Sigma}_{\mathbf{Z}}$ (that is, $\mathbf{Z} \sim \mathcal{N}\left(\boldsymbol{\mu}_{\mathbf{Z}}, \boldsymbol{\Sigma}_{\mathbf{Z}}\right)$ ), $\mathcal{A}$ is a non-random $n \times n$ matrix function of $t, \gamma$ is a non-random $n$-dimensional column vector function of $t$, and $\mathcal{H}$ is a non-random $n \times m$ matrix function of $t$. The corresponding system of stochastic differential equations has the form

$$
d \mathbf{X}(t)=[\mathcal{A}(t) \mathbf{X}(t)+\boldsymbol{\xi}(t)] d t+\mathcal{F}(t) d \mathbf{W}(t), \quad \mathbf{X}(0)=\mathbf{X}_{0},
$$

where $\boldsymbol{\xi}$ is a non-random $n$-dimensional column vector function of $t, \mathcal{F}$ is a non-random $n \times l$ matrix function of $t$, and $\mathbf{W}(t)=\left(W_{1}(t), W_{2}(t), \ldots, W_{l}(t)\right)^{T}$ is a $l$-vector standard Wiener process.

Let $\boldsymbol{\Phi}(t)$ be the solution of the associated deterministic initial value problem

$$
\frac{d \boldsymbol{\Phi}(t)}{d t}=\mathcal{A}(t) \boldsymbol{\Phi}(t), \quad \boldsymbol{\Phi}(0)=\mathcal{I}_{n},
$$

where $\mathcal{I}_{n}$ is the $n \times n$ identity matrix. Then the conditions for the pointwise equivalence between the system of random differential equations (3.9) and the system of stochastic differential equations (3.10) are stated in the following theorem (see [17] for proofs).

Theorem 3.4. If functions $\boldsymbol{\xi}, \mathcal{F}, \gamma, \mathcal{H}, \boldsymbol{\mu}_{\mathbf{Z}}$ and $\boldsymbol{\Sigma}_{\mathbf{Z}}$ satisfy the following two equalities

$$
\int_{0}^{t} \boldsymbol{\Phi}^{-1}(s)\left(\gamma(s)+\mathcal{H}(s) \boldsymbol{\mu}_{\mathbf{Z}}\right) d s=\int_{0}^{t} \boldsymbol{\Phi}^{-1}(s) \boldsymbol{\xi}(s) d s,
$$

and

$$
\left[\int_{0}^{t} \boldsymbol{\Phi}^{-1}(s) \mathcal{H}(s) d s\right] \boldsymbol{\Sigma}_{\mathbf{Z}}\left[\int_{0}^{t} \boldsymbol{\Phi}^{-1}(s) \mathcal{H}(s) d s\right]^{T}=\int_{0}^{t} \boldsymbol{\Phi}^{-1}(s) \mathcal{F}(s)\left(\boldsymbol{\Phi}^{-1}(s) \mathcal{F}(s)\right)^{T} d s,
$$

then the system of random differential equations (3.9) and the system of stochastic differential equations (3.10) are pointwise equivalent.

Conversion of Systems of RDEs to Systems of SDEs Based on the equivalence conditions (3.12) and (3.13), the specific form of the corresponding pointwise equivalent system of SDEs can be given in terms of parameters for a given system of RDEs. To do that, we assume that the following matrix function

$$
\boldsymbol{\Pi}_{p}(t)=\left[\int_{0}^{t} \boldsymbol{\Phi}^{-1}(s) \mathcal{H}(s) d s\right] \boldsymbol{\Sigma}_{\mathbf{Z}}\left[\int_{0}^{t} \boldsymbol{\Phi}^{-1}(s) \mathcal{H}(s) d s\right]^{T}
$$

has the property that the matrix function $\boldsymbol{\Phi}(t) \dot{\boldsymbol{\Pi}}_{p}(t)(\boldsymbol{\Phi}(t))^{T}$ is positive-semidefinite for any $t \geq 0$. Then the corresponding pointwise equivalent system of SDEs for the system of RDEs (3.9) is given by

$$
d \mathbf{X}(t)=\left[\mathcal{A}(t) \mathbf{X}(t)+\gamma(t)+\mathcal{H}(t) \boldsymbol{\mu}_{\mathbf{Z}}\right] d t+\mathcal{F}(t) d \mathbf{W}(t), \quad \mathbf{X}(0)=\mathbf{X}_{0},
$$

where the matrix function $\mathcal{F}$ satisfies

$$
\mathcal{F}(t) \mathcal{F}^{T}(t)=\boldsymbol{\Phi}(t) \dot{\boldsymbol{\Pi}}_{p}(t)(\boldsymbol{\Phi}(t))^{T} .
$$

Conversion of Systems of SDEs to Systems of RDEs The equivalence conditions (3.12) and (3.13) imply that there are many different choices for the equivalent system of RDEs. One of the simple 
choices for the corresponding pointwise equivalent system of RDEs for the system of SDEs (3.10) is given by

$$
\frac{d \mathbf{x}\left(t ; \mathbf{X}_{0}, \mathbf{Z}\right)}{d t}=\mathcal{A}(t) \mathbf{x}\left(t ; \mathbf{X}_{0}, \mathbf{Z}\right)+\boldsymbol{\xi}(t)+\boldsymbol{\Phi}(t) \dot{\Lambda}(t) \mathbf{Z}, \quad \mathbf{x}\left(0 ; \mathbf{X}_{0}, \mathbf{Z}\right)=\mathbf{X}_{0},
$$

where $\mathbf{Z}$ is an $n$-dimensional random vector with $\mathbf{Z} \sim \mathcal{N}\left(\mathbf{0}, \mathcal{I}_{n}\right)$, and the matrix function $\boldsymbol{\Lambda}$ satisfies the equality

$$
\boldsymbol{\Lambda}(t)(\boldsymbol{\Lambda}(t))^{T}=\int_{0}^{t} \boldsymbol{\Phi}^{-1}(s) \mathcal{F}(s)\left(\boldsymbol{\Phi}^{-1}(s) \mathcal{F}(s)\right)^{T} d s .
$$

\subsection{Pointwise Equivalent Nonlinear Differential Equations}

The discussions in the above three subsections imply that if a nonlinear stochastic differential equation (or a system of nonlinear stochastic differential equations) can be reduced to one of the forms in (3.2) or (3.6) (or the form (3.10)) by some invertible transformation, then one can find its corresponding pointwise equivalent random differential equation. The converse is true for random differential equations.

The following two theorems (given in [33, Section 4.1]) show that there is a large class of nonlinear stochastic differential equations that can be reduced to linear stochastic differential equations after some invertible transformation and thus are pertinent to our discussions here.

Theorem 3.5. Consider the stochastic differential equation

$$
d X(t)=g(t, X(t)) d t+\sigma(t, X(t)) d W(t),
$$

where $g$ and $\sigma$ are non-random functions of $x$ and $t$. If the equality

$$
\frac{\partial}{\partial x}\left\{\sigma(t, x)\left[\frac{1}{\sigma^{2}(t, x)} \frac{\partial \sigma}{\partial t}(t, x)-\frac{\partial}{\partial x}\left(\frac{g}{\sigma}\right)(t, x)+\frac{1}{2} \frac{\partial^{2} \sigma}{\partial x^{2}}(t, x)\right]\right\}=0
$$

holds, then (3.14) can be reduced to the linear stochastic differential equation

$$
d Y(t)=\bar{g}(t) d t+\bar{\sigma}(t) d W(t) .
$$

Here $\bar{\sigma}$ is some deterministic function determined from

$$
\bar{\sigma}^{\prime}(t)=\bar{\sigma}(t) \sigma(t, x)\left[\frac{1}{\sigma^{2}(t, x)} \frac{\partial \sigma}{\partial t}(t, x)-\frac{\partial}{\partial x}\left(\frac{g}{\sigma}\right)(t, x)+\frac{1}{2} \frac{\partial^{2} \sigma}{\partial x^{2}}(t, x)\right],
$$

and $\bar{g}$ is some deterministic function given by

$$
\bar{g}(t)=\frac{\partial h}{\partial t}(t, x)+\frac{\partial h}{\partial x}(t, x) g(t, x)+\frac{1}{2} \frac{\partial^{2} h}{\partial x^{2}}(t, x) \sigma^{2}(t, x)
$$

with $h$ being some smooth invertible function computed from

$$
\frac{\partial h}{\partial x}(t, x)=\frac{\bar{\sigma}(t)}{\sigma(t, x)} .
$$

In addition, the relationship between $Y(t)$ and $X(t)$ is given by $Y(t)=h(t, X(t))$.

Theorem 3.6. The autonomous stochastic differential equation

$$
d X(t)=g(X(t)) d t+\sigma(X(t)) d W(t)
$$

can be reduced to the following linear stochastic differential equation

$$
d Y(t)=\left(\lambda_{0}+\lambda_{1} Y(t)\right) d t+\left(\nu_{0}+\nu_{1} Y(t)\right) d W(t)
$$


if and only if

$$
\psi^{\prime}(x)=0 \text { or }\left(\frac{\left(\sigma \psi^{\prime}\right)^{\prime}}{\psi^{\prime}}\right)^{\prime}(x)=0
$$

Here $g$ and $\sigma$ are non-random functions of $x, \lambda_{0}, \lambda_{1}, \nu_{0}$ and $\nu_{1}$ are some constants, $\psi(x)=\frac{g(x)}{\sigma(x)}-\frac{1}{2} \sigma^{\prime}(x)$, and $Y(t)=h(X(t))$, where $h$ is some invertible transformation.

If the latter part of $(3.15)$ is satisfied, then we set $\nu_{1}=-\frac{\left(\sigma \psi^{\prime}\right)^{\prime}}{\psi^{\prime}}$ and choose

$$
h(x)= \begin{cases}c_{1} \exp \left(\nu_{1} \int_{a}^{x} \frac{1}{\sigma(\tau)} d \tau\right), & \text { if } \nu_{1} \neq 0 \\ \nu_{0} \int_{a}^{x} \frac{1}{\sigma(\tau)} d \tau+c_{0}, & \text { if } \nu_{1}=0,\end{cases}
$$

where $c_{0}$ and $c_{1}$ are some constants.

Based on the equivalence results in Sections 3.1-3.3 as well as the results presented in Theorem 3.5 and 3.6, one can derive the corresponding pointwise equivalent RDEs for a large class of nonlinear SDEs. Specifically, in [17] several different nonlinear examples were given to illustrate this transformation method to find the corresponding equivalent SDE/RDE and RDE/SDE. These examples include the popular Verhulst-Pearl logistic growth model $\frac{d x}{d t}=r x\left(1-\frac{x}{K}\right)$ and the general growth model $\frac{d x}{d t}=$ $\left(a_{0}(t)-a_{1}(t) \ln x\right) x$ of which the standard Gompertz growth model $\frac{d x}{d t}=r(\ln b-\ln x) x$ is a special case.

\subsection{Remarks on Relationships Between the FPPS and GRDPS Models}

To establish the pointwise equivalence between SDEs and RDEs in the above subsections, we assume a normal distribution on each component of $\mathbf{Z}$ (i.e., $Z_{j} \sim \mathcal{N}\left(\mu_{j}, \sigma_{j}^{2}\right)$ ) for the RDE in Sections 3.1 and 3.2 (and impose a multivariate normal distribution on $\mathbf{Z}$ in Section 3.3). However, this normal assumption is not completely reasonable in many applications because the growth rate may become negative for many individuals (which results in the size classes having non-negligible probability of being negative in a finite time period) in the population when the variance $\sigma_{j}^{2}$ is sufficiently large relative to $\mu_{j}$. A standard approach in practice to remedy this problem is to impose a truncated normal distribution instead of a normal distribution; that is, we restrict $Z_{j}$ in some reasonable range. We also observe that the corresponding stochastic formulation also can lead to the size having non-negligible probability being negative when the diffusion dominates the drift because $W(t) \sim \mathcal{N}(0, t)$ for any fixed $t$ and hence decreases in size are possible. One way to remedy this situation is to set $X(t)=0$ if $X(t) \leq 0$. Thus, if $\sigma_{j}$ is sufficiently large relative to $\mu_{j}$, then we may obtain different probability density functions for the solutions of the RDE and SDE after we have made these different modifications to each. The same anomalies (demonstrated in [14] with some computational examples) hold for the solutions of the associated FPPS and GRDPS models themselves when we impose zero-flux boundary conditions (which is equivalent to setting $X(t)=0$ if $X(t) \leq 0$ for the SDE in the sense that both are used to keep individuals in the system) in the FPPS model and put constraints on each component of $\mathbf{Z}$ in the GRDPS model.

However, for the case where the truncated normal distribution can provide a good approximation to the normal distribution (i.e., for the case where $\sigma_{j}$ is sufficiently small relative to $\mu_{j}$ ), the GRDPS model and the corresponding FPPS model can, with appropriate boundary and initial conditions as well as properly chosen parameters based on pointwise equivalence results between the SDE and RDE, yield quite similar solutions. This is predicated by our analysis of the SDE and the RDE and their resulting FPPS and GRDPS models, and was also demonstrated in [14] with several computational examples. It is well documented (e.g., see $[26,36]$ and the references therein) that there exist significant difficulties in numerically solving FPPS models when the drift dominates the diffusion (the case of primary interest in 
many situations) even for the scalar case. However, as mentioned above the GRDPS models are readily solved rapidly with so-called "embarrassing parallel" computational methods $[16,65]$, where the model governed for each subpopulation could be solved via the methods of characteristic (when dimensions are relative small, such as less than or equal to three) or some other well-established numerical methods (e.g., $[2,4]$ and the references therein). Hence, the corresponding GRDPS model could be used as an alternative method that can be fast and efficient in numerically solving for the pointwise equivalent distributions in the FPPS model.

\section{Concluding Remarks}

In this paper we gave a review of two conceptually different approaches that have been used in the literature to model growth uncertainty in a physiologically structured population. We also give a summary of the relationship between these two approaches as well as the relationship between their resulting physiologically structured population models. A more general approach to model the growth uncertainty would be to combine the two approaches that we considered in this paper. That is, the growth processes of individuals would be characterized by the system of differential equations

$$
d \mathbf{X}(t)=\mathbf{g}(t, \mathbf{X}(t) ; \mathbf{Z}) d t+\boldsymbol{\sigma}(t, \mathbf{X}(t) ; \mathbf{Z}) d \mathbf{W}(t), \quad \mathbf{X}(0)=\mathbf{X}_{0} .
$$

Such a formulation was used in [38] to model the dynamics of heterogeneous cell populations. In some cases, the growth processes of individuals may be driven by a much more complicated dynamical system such as one given by

$$
d \mathbf{X}(t)=\mathbf{g}(t, \mathbf{X}(t) ; \mathbf{Z}(t)) d t+\boldsymbol{\sigma}(t, \mathbf{X}(t) ; \mathbf{Z}(t)) d \mathbf{W}(t), \quad \mathbf{X}(0)=\mathbf{X}_{0},
$$

where $\mathbf{Z}(t)$ is a stochastic process (with either discrete or continuous state space) which may be dependent on $\mathbf{X}(t)$. In general, it is difficult if not impossible to obtain an equation for the resulting probability density function of $\mathbf{X}(t)$, especially in the case where $\mathbf{Z}(t)$ is dependent on $\mathbf{X}(t)$.

In reality, mortality and reproduction rates may also be random. Thus, it would be interesting (and as yet an open question) to know how to correctly incorporate these uncertainties into the existing dynamical systems (e.g., the GRDPS models or FPPS models), and investigate the relationship between them. In addition, it would be interesting to know the stochastic processes with which those nonlinear structured population models (e.g., a size structured population model with growth rate depending on the total number of population) are associated.

Even though the FPPS and GRDPS models have been proven quite useful in specific applications, they are still less explored in the literature in terms of either computational or theoretical analysis, especially in the area of optimal control studies. The investigation of control studies for these systems is important not only conceptually but also for applications. For example, it is well known that growth rates of marine species such as shrimp depend on a number of environmental factors such as temperature, salinity level and oxygen level. Hence, it is important to know how to optimally control these factors in order to produce maximum growth, especially in commercial efforts (see $[13,30]$ and the references therein).

Acknowledgements. This research was supported in part by Grant Number NIAID R01AI071915-09 from the National Institute of Allergy and Infectious Disease, in part by the U.S. Air Force Office of Scientific Research under grant number FA9550-09-1-0226 and in part by the National Institute on Alcohol Abuse and Alcoholism under a subcontract from the Research Foundation for Mental Hygiene.

\section{References}

[1] A.S. Ackleh, H.T. Banks, K. Deng. A finite difference approximation for a coupled system of nonlinear size-structured populations. Nonlinear Analysis, 50 (2002), 727-748.

[2] A.S. Ackleh, K. Ito. An implicit finite-difference scheme for the nonlinear size-structured population model. Numer. Funct. Anal. Optim. 18 (1997), 65-884. 
[3] A.S. Ackleh, K. Deng. A monotone approximation for the nonautonomous size-structured population model. Quart. Appl. Math., 57 (1999), 261-267.

[4] O. Angulo, J.C. López-Marcos, Numerical integration of fully nonlinear size-structured population models. Applied Numerical Mathematics, 50 (2004), 291-327.

[5] H.T. Banks. A Functional Analysis Framework for Modeling, Estimation and Control in Science and Engineering, CRC Press/Taylor and Frances Publishing, Boca Raton, FL, June, 2012, (258 pages).

[6] H.T. Banks, K.L. Bihari. Modelling and estimating uncertainty in parameter estimation. Inverse Problems, 17 (2001), 95-111.

[7] H.T. Banks, V.A. Bokil, S. Hu, A.K. Dhar, R.A. Bullis, C.L. Browdy, F.C.T. Allnutt. Modeling shrimp biomass and viral infection for production of biological countermeasures CRSC-TR05-45, NCSU, December, 2005; Mathematical Biosciences and Engineering, 3 (2006), 635-660.

[8] H.T. Banks, L.W. Botsford, F. Kappel, C. Wang. Modeling and estimation in size structured population models. LCDS-CCS Report 87-13, Brown University; Proceedings 2nd Course on Mathematical Ecology, (Trieste, December 8-12, 1986) World Press, Singapore, 1988, 521-541.

[9] H.T. Banks, F. Charles, M. Doumic, K.L. Sutton, W.C. Thompson. Label structured cell proliferation models. Appl. Math. Letters, 23 (2010), 1412-1415.

[10] H.T. Banks, J.L. Davis. A comparison of approximation methods for the estimation of probability distributions on parameters. CRSC-TR05-38, October, 2005; Applied Numerical Mathematics, 57 (2007), 753-777.

[11] H.T. Banks, J.L. Davis. Quantifying uncertainty in the estimation of probability distributions. CRSC-TR07-21, December, 2007; Math. Biosci. Engr., 5 (2008), 647-667.

[12] H.T. Banks, J.L. Davis, S.L. Ernstberger, S. Hu, E. Artimovich, A.K. Dhar, C.L. Browdy. A comparison of probabilistic and stochastic differential equations in modeling growth uncertainty and variability. CRSC-TR08-03, NCSU, February, 2008; Journal of Biological Dynamics, 3 (2009), 130-148.

[13] H.T. Banks, J.L. Davis, S.L. Ernstberger, S. Hu, E. Artimovich, A.K. Dhar. Experimental design and estimation of growth rate distributions in size-structured shrimp populations. CRSC-TR08-20, NCSU, November, 2008; Inverse Problems, 25 (2009), 095003(28pp).

[14] H.T. Banks, J.L. Davis, S. Hu. A computational comparison of alternatives to including uncertainty in structured population models. CRSC-TR09-14, June, 2009; Three Decades of Progress in Systems and Control, X. Hu, U. Jonsson, B. Wahlberg, B. Ghosh (Eds.), Springer, 2010, 19-33.

[15] H.T. Banks, B.G. Fitzpatrick. Estimation of growth rate distributions in size structured population models. Quarterly of Applied Mathematics, 49 (1991), 215-235.

[16] H.T. Banks, B.G. Fitzpatrick, L.K. Potter, Y. Zhang. Estimation of probability distributions for individual parameters using aggregate population data. CRSC-TR98-6, NCSU, January, 1998; Stochastic Analysis, Control, Optimization and Applications, (Edited by W. McEneaney, G. Yin and Q. Zhang), Birkhauser, Boston, 1998, 353-371.

[17] H.T. Banks, S. Hu. Nonlinear stochastic Markov processes and modeling uncertainty in populations. CRSC-TR11-02, NCSU, January, 2011; Mathematical Bioscience and Engineering, 9 (2012), 1-25.

[18] H.T. Banks, F. Kappel. Transformation semigroups and $L^{1}$-approximation for size- structured population models. Semigroup Forum, 38 (1989), 141-155.

[19] H.T. Banks, F. Kappel, C. Wang. Weak solutions and differentiability for size-structured population models. International Series of Numerical Mathematics, 100 (1991), 35-50.

[20] H.T Banks, G.A. Pinter. A probabilistic multiscale approach to hysteresis in shear wave propagation in biotissue, CRSC-TR04-03, January, 2004; SIAM J. Multiscale Modeling and Simulation, 3 (2005), 395-412.

[21] H.T. Banks, K.L. Sutton, W.C. Thompson, G. Bocharov, M. Doumic, T. Schenkel, J. Argilaguet, S. Giest, C. Peligero, A. Meyerhans. A new model for the estimation of cell proliferation dynamics using CFSE data. Center for Research in Scientific Computation Technical Report CRSC-TR11-05, NCSU, July, 2011; J. Immunological Methods, 373 (2011), 143-160.

[22] H.T. Banks, K.L. Sutton, W.C. Thompson, G. Bocharov, D. Roose, T. Schenkel, A. Meyerhans. Estimation of cell proliferation dynamics using CFSE data, CRSC-TR09-17, NCSU, August, 2009; Bull. Math. Biol. 70 (2011), 116-150; doi:10.1007/s11538-010-9524-5.

[23] H.T. Banks, W.C. Thompson. Mathematical models of dividing cell populations: Application to CFSE data. CRSCTR12-10, N. C. State University, Raleigh, NC, April, 2012; Journal on Mathematical Modelling of Natural Phenomena, to appear.

[24] H.T. Banks, W.C. Thompson. A division-dependent compartmental model with cyton and intracellular label dynamics. CRSC-TR12-12, N. C. State University, Raleigh, NC, May, 2012; Intl. J. of Pure and Applied Math., 77 (2012), 119-147.

[25] H.T. Banks, W.C. Thompson, C. Peligero, S. Giest, J. Argilaguet, A. Meyerhans. A compartmental model for computing cell numbers in CFSE-based lymphocyte proliferation assays, CRSC-TR12-03, NCSU, January, 2012; Math. Biosci. Engr., to appear.

[26] H.T. Banks, H.T. Tran, D.E. Woodward. Estimation of variable coefficients in the Fokker-Planck equations using moving node finite elements. SIAM J. Numer. Anal., 30 (1993), 1574-1602.

[27] G.I. Bell, E.C. Anderson. Cell growth and division I. a mathematical model with applications to cell volume distributions in mammalian suspension cultures. Biophysical Journal, 7 (1967), 329-351.

[28] A. Calsina, J. Saldana. A model of physiologically structured population dynamics with a nonlinear individual growth rate. Journal of Mathematical Biology, 33 (1995), 335-364. 
[29] G. Casella, R.L. Berger. Statistical Inference. Duxbury, California, 2002.

[30] F.L. Castille, T.M. Samocha, A.L. Lawrence, H. He, P. Frelier, F. Jaenike. Variability in growth and survival of early postlarval shrimp (Penaeus vannamei Boone 1931) Aquaculture, 113 (1993), 65-81.

[31] J. Chu, A. Ducrot, P. Magal, S. Ruan. Hopf bifurcation in a size-structured population dynamic model with random growth. J. Differential Equations, 247 (2009), 956-1000.

[32] J.M. Cushing. An Introduction to Structured Population Dynamics. CMB-NSF Regional Conference Series in Applied Mathematics, SIAM, Philadelphia, PA, 1998.

[33] T.C. Gard. Introduction to Stochastic Differential Equations. Marcel Dekker, New York, 1988.

[34] C.W. Gardiner. Handbook of Stochastic Methods for Physics, Chemistry and the Natural Sciences. Springer-Verlag, Berlin, 1983.

[35] M. Gyllenberg, G.F. Webb. A nonlinear structured population model of tumor growth with quiescence. J. Math. Biol., 28 (1990), 671-694.

[36] G.W. Harrison. Numerical solution of the Fokker-Planck equation using moving finite elements. Numerical Methods for Partial Differential Equations, 4 (1988), 219-232.

[37] J. Hasenauer, D. Schittler, F. Allgöer. A computational model for proliferation dynamics of division- and labelstructured populations. February, 2012, preprint.

[38] J. Hasenauer, S. Waldherr, M. Doszczak, P. Scheurich, N. Radde, F. Allgöer. Analysis of heterogeneous cell populations: a density-based modeling and identification framework. J. Process Control, 21 (2011), 1417-1425.

[39] K. Huang. Statistical Mechanics. J. Wiley \& Sons, New York, NY, 1963.

[40] M. Iannelli. Mathematical Theory of Age-Structured Population Dynamics. Applied Math. Monographs, CNR, Giardini Editori e Stampatori, Pisa, 1995.

[41] M. Kimura. Process leading to quasi-fixation of genes in natural populations due to random fluctuation of selection intensities. Genetics, 39 (1954), 280-295.

[42] F. Klebaner. Introduction to Stochastic Calculus with Applications. 2nd ed., Imperial College Press, London, 2006.

[43] I. Karatzas, S.E. Shreve, Brownian Motion and Stochastic Calculus, Second Edition, Springer, New York, 1991.

[44] T. Luzyanina, D. Roose, G. Bocharov. Distributed parameter identification for a label-structured cell population dynamics model using CFSE histogram time-series data. J. Math. Biol., 59 (2009), 581-603.

[45] T. Luzyanina, M. Mrusek, J.T. Edwards, D. Roose, S. Ehl, G. Bocharov. Computational analysis of CFSE proliferation assay. J. Math. Biol., 54 (2007), 57-89.

[46] T. Luzyanina, D. Roose, T. Schenkel, M. Sester, S. Ehl, A. Meyerhans, G. Bocharov. Numerical modelling of labelstructured cell population growth using CFSE distribution data. Theoretical Biology and Medical Modelling, 4(2007), Published Online.

[47] A.G. McKendrick. Applications of mathematics to medical problems. Proceedings of the Edinburgh Mathematical Society, 44 (1926), 98-130.

[48] J.A.J. Metz, E.O. Diekmann, The Dynamics of Physiologically Structured Populations. Lecture Notes in Biomathematics, Vol. 68, Springer, Heidelberg, 1986.

[49] J.E. Moyal. Stochastic processes and statistical physics. Journal of the Royal Statistical Society. Series B (Methodological), 11 (1949), 150-210.

[50] Yu. V. Prohorov. Convergence of random processes and limit theorems in probability theory. Theor. Prob. Appl., 1 (1956), 157-214.

[51] B. Oksendal. Stochastic Differentail Equations. 5th edition, Springer, Berlin, 2000.

[52] A. Okubo. Diffusion and Ecological Problems: Mathematical Models. Biomathematics, 10 (1980), Springer-Verlag, Berlin.

[53] G. Oster,Y. Takahashi. Models for age-specific interactions in a periodic environment. Ecological Monographs, 44 (1974), 483-501.

[54] B. Perthame. Transport Equations in Biology. Birkhauser Verlag, Basel, 2007.

[55] R. Rudnicki. Models of population dynamics and genetics. From Genetics To Mathematics, (edited by M. Lachowicz and J. Miekisz), World Scientific, Singapore, 2009, 103-148.

[56] H. Risken. The Fokker-Planck Equation: Methods of Solution and Applications. Springer, New York, 1996.

[57] D. Schittler, J. Hasenauer, F. Allgöer. A generalized population model for cell proliferation: Integrating division numbers and label dynamics. Proceedings of Eighth International Workshop on Computational Systems Biology (WCSB 2011), June 2011, Zurich, Switzerland, 165-168.

[58] D. Schittler, J. Hasenauer, F. Allgöer. A model for proliferating cell populations that accounts for cell types. Proc. of 9th International Workshop on Computational Systems Biology, 2012, 84-87.

[59] J. Sinko, W. Streifer. A new model for age-size structure of a population. Ecology, 48 (1967), 910-918.

[60] T.T. Soong. Random Differential Equations in Science and Engineering. Academic Press, New York and London, 1973.

[61] T.T. Soong, S.N. Chuang. Solutions of a class of random differential equations. SIAM J. Appl. Math., 24 (1973), 449-459.

[62] W.C. Thompson. Partial Differential Equation Modeling of Flow Cytometry Data from CFSE-based Proliferation Assays. Ph.D. Dissertation, North Carolina State University, December, 2011.

[63] H. Von Foerster. Some remarks on changing populations. The Kinetics of Cellular Proliferation, F. Stohlman, Jr. (ed.), Grune and Stratton, New York, 1959. 
[64] G.F. Webb. Theory of Nonlinear Age-dependent Population Dynamics. Marcel Dekker, New York, 1985.

[65] A.Y. Weiße, R.H. Middleton, W. Huisinga. Quantifying uncertainty, variability and likelihood for ordinary differential equation models. BMC Syst. Bio., 4 (144), 2010.

[66] G.H. Weiss Equation for the age structure of growing populations. Bull. Math. Biophy., 30 (1968), $427-435$.

[67] http://en.wikipedia.org/wiki/Probability_density_function. 